DOI: $10.15393 / \mathrm{j} 3 . \operatorname{art} .2018 .5290$

UDC 517.545

S. R. NASYROV

\title{
FAMILIES OF ELLIPTIC FUNCTIONS AND UNIFORMIZATION OF COMPLEX TORI WITH A UNIQUE POINT OVER INFINITY
}

\begin{abstract}
We investigate the problem of describing a one-parametric family of elliptic functions which uniformizes a given family of ramified coverings of the Riemann sphere with maximal possible ramification over infinity. We find a PDE for the family of functions and use it to deduce a system of ODEs for their critical points.
\end{abstract}

Key words: Riemann surface, complex torus, elliptic function, uniformization

2010 Mathematical Subject Classification: 30F10, 32G15

1. Introduction. Let $S$ be an $n$-sheeted compact Riemann surface over the Riemann sphere $\overline{\mathbb{C}}$ with a unique point over infinity of multiplicity $n-1$. We will mainly consider the case when the genus $\rho$ of $S$ equals 1 , i. e., $S$ is a complex torus; however, in Section 2, following [12], we also shortly describe the simply-connected case $(\rho=0)$.

If $\rho=0$, then, by the well-known Koebe-Poincare uniformization theorem, there exists a polynomial $f$ which maps $\overline{\mathbb{C}}$ onto $S$ conformally. In the case $\rho=1$ the surface $S$ can be uniformized by an elliptic function, i. e., there exists a two-periodic meromorphic function $f$ with periods $\omega_{1}$ and $\omega_{2}$ such that the factor-mapping $\tilde{f}: \mathbb{C} / \Omega \rightarrow S$ is a conformal isomorphism. Here $\Omega$ is the lattice generated by $\omega_{1}$ and $\omega_{2}$.

Denote the critical points of $f$ by $a_{j}, 1 \leqslant j \leqslant N$. Since $S$ is given, we know the critical values $A_{j}=f\left(a_{j}\right), 1 \leqslant j \leqslant N$, and their multiplicities $m_{j}$. The problem of uniformization of $S$ is reduced, in essence, to finding the values $a_{j}, 1 \leqslant j \leqslant N$.

The system

$$
f\left(a_{j}\right)=A_{j}, \quad 1 \leqslant j \leqslant N,
$$

is a system of non-linear equations with respect to $a_{j}$, with certain difficulties in solving. Hurwitz [3] first noted that, critical values are not defined (C) Petrozavodsk State University, 2018 
uniquely by given $A_{j}$ and multiplicities $m_{j}$. He posed the problem of the number of non-equivalent coverings with the same branch data.

The Hurwitz problem. Let $\rho \in \mathbb{Z}_{+}$, naturals numbers $n_{j}^{(k)}$, $j=1, \ldots, h_{k}, k=0, \ldots, m$, satisfy

$$
\begin{gathered}
\sum_{j=1}^{h_{k}} n_{j}^{(k)}=n, \quad k=0, \ldots, m, \\
\sum_{k=0}^{m} \sum_{j=1}^{h_{k}}\left(n_{j}^{(k)}-1\right)=(2-2 \rho) n-2,
\end{gathered}
$$

and a finite set $\mathfrak{T}=\left\{T_{0}, T_{1}, \ldots, T_{m}\right\} \subset \overline{\mathbb{C}}$ be given. Find how many nonequivalent compact Riemann surfaces over $\overline{\mathbb{C}}$ of fixed genus $\rho$ exist, such that their branch points lie over $\mathfrak{T}$ and for every $k$ there exist exactly $h_{k}$ points of multiplicities $n_{1}^{(k)}-1, \ldots, n_{h_{k}}^{(k)}-1$, lying over $T_{k}, k=0, \ldots, m$.

The Hurwitz problem and its generalizations were investigated, in particular, in [3-10,14]. The obtained results show that (1) can have multiple solutions, and distinct solutions can correspond to non-equivalent coverings of the Riemann sphere. Therefore, even if we solve system (??) and find several solutions, we have to choose the one that matches the given surface $S$.

In the paper we propose an approximate method of finding $a_{j}$. Its essence is in the following. Consider the space $\Sigma$ of all $n$-sheeted Riemann surfaces of genus $\rho$, which are ramified covering of $\mathbb{C}$, with the same branch data as $S$. The topology on $\Sigma$ is induced by the Caratheodory kernel convergence (see, e.g., [10]). Let us include $S$ into a smooth one-parametric family $S(t), 0 \leqslant t \leqslant 1$, such that $S(1)=S$, and the uniformizing function $f(z, t)$ for $S(t)$ is known for $t=0$; therefore, we know its critical points $a_{j}^{0}$. Smoothness of the family means that projections $A_{j}(t)$ of branch points depend smoothly on $t$.

We describe the movement of $a_{j}(t)$ with the help of a system of ODEs (Theorem 7). Solving the Cauchy problem for the system with the initial data $\left(a_{j}^{0}\right)$ we obtain dependences $a_{j}(t), 0 \leqslant t \leqslant 1$, and the final values $(t=1)$ give the desired critical values $a_{j}$ for $S$. Note that in the case $\rho=1$ we need to determine, additionally, the parameter $\omega_{2} / \omega_{1}$ that depends on $t$, called the module of the complex torus $S$.

To obtain the system of ODEs mentioned above, we deduce the PDE

$$
\frac{\partial f(z, t)}{\partial t}=h(z, t) \frac{\partial f(z, t)}{\partial z}
$$


which the family $f(z, t)$ satisfies to (Theorem 6$)$. The idea of using oneparametric families of analytic functions and equations of such type belongs to Loewner who offered his famous parametric method in the theory of univalent functions; a more general equation of the form (2) was suggested by Kufarev (see, e. g., [2]).

Note that in [13] we investigated one-parametric families of elliptic functions with simple critical points. Here we study the case of arbitrary multiplicities.

2. The simply-connected case. The case $\rho=0$ is described in [11]. In [12] a more general case is investigated: when $f(z, t)$ is a family of rational functions. Here we formulate the main results from [12] specifying them for the case of polynomials.

Consider a smooth one-parametric family of polynomials

$$
P(z, t)=\int_{a_{1}}^{z} \prod_{j=1}^{N-1}\left(\zeta-a_{j}\right)^{m_{j}-1} d \zeta=\frac{z^{n}}{n}-\sum_{j=1}^{N-1} m_{j} a_{j} \frac{z^{n-1}}{n-1}+\ldots
$$

$0 \leqslant t \leqslant 1$, where $a_{j}=a_{j}(t)$ are pairwise distinct points of the complex plane, $N \geqslant 3, \quad m_{j}-1 \in \mathbb{N}, \quad \sum_{j=1}^{N-1}\left(m_{j}-1\right)=n-1$.

We will also assume that

$$
\sum_{j=1}^{N-1} m_{j} a_{j}=0
$$

This condition can be fulfilled by a shift of the $z$-plane.

Denote

$$
H_{l}(z)=\frac{1}{\prod_{j=1, j \neq l}^{N-1}\left(z-a_{j}\right)^{m_{j}}} .
$$

Let $P_{j, m_{j}-1}(z, t)$ be the Taylor's polynomial of $H_{l}(z)$ of degree $\left(m_{j}-1\right)$ at the point $a_{j}$ and $A_{j}(t)=f\left(a_{j}(t), t\right), 1 \leqslant j \leqslant N-1$. From (3) it follows that $A_{1}(t) \equiv 0$. This does not restrict generality because the condition can be provided by a shift in the plane.

Theorem 1. The family $P(z, t)$ satisfies the PDE

$$
\dot{P}(z, t)=h(z, t) P^{\prime}(z, t), \quad z \in \mathbb{C}, 0 \leqslant t \leqslant 1,
$$


where

$$
h(z, t)=\sum_{j=2}^{N-1} \frac{P_{j, m_{j}-1}(z, t)}{\left(z-a_{j}(t)\right)^{m_{j}}} \dot{A}_{j}(t) .
$$

Here and further the dot above a letter denotes differentiation with respect to $t$ (the partial derivative if function depends on $z$ and $t$, and the ordinary one for function of the only variable $t$ ), and the prime means the derivative by $z$.

Theorem 2. The critical points $a_{j}(t)$ satisfy the system of ODEs

$$
\dot{a}_{l}=\frac{H_{l}^{\left(m_{l}-1\right)}\left(a_{l}\right)}{\left(m_{l}-1\right) !} \dot{A}_{l}+\sum_{k=2, k \neq l}^{N-2} \frac{G_{k l}^{\left(m_{k}-2\right)}\left(a_{k}\right)}{\left(m_{k}-2\right) !} \dot{A}_{k}, \quad 1 \leqslant l \leqslant N-1,
$$

where $G_{k l}(z)=H_{k}(x) /\left(z-a_{l}\right)$.

Note that (4) allows to find the critical points of the polynomial, uniformizing a given simply-connected Riemann surface, quickly and with a good accuracy. While solving (4), we obtain the whole family of polynomials uniformizing a definite family of surfaces. If we know a solution for a set of $A_{j}$ with fixed branch data, then we can obtain other solutions moving $A_{j}$ along various closed curves.

We can also consider (4) as variational formulae for polynomials, expressing variations of critical points through variations of critical values. The formulae can be useful for solving some extremal problems of geometric function theory connected with polynomials.

3. Preliminary results on elliptic functions. Now we will investigate smooth one-parametric families of $n$-sheeted complex tori. We assume that for every torus there exists a unique point lying over infinity and connecting all its sheets. The case when all other branch points are simple was studied in [13]; here we investigate the case of arbitrary multiplicities.

First we recall some classical results of the theory of elliptic functions (see, e.g., [1]).

A non-constant meromorphic in $\mathbb{C}$ function is called elliptic, if it has two periods $\omega_{1}$ and $\omega_{2}$, linearly independent over $\mathbb{R}$ and generating the lattice $\Omega$ of all its periods. Without loss of generality we assume that

$$
\operatorname{Im} \frac{\omega_{2}}{\omega_{1}}>0
$$


The period parallelogram is a parallelogram with vertex at some point generated by vectors $\omega_{1}$ and $\omega_{2}$

In every period parallelogram a non-constant elliptic function takes every value the same number of times (the Liouville's theorem); the number is called the order of the elliptic function.

Let $a_{1}, \ldots, a_{n}$ be zeroes of a non-constant elliptic function and $b_{1}, \ldots, b_{n}$ be its poles lying in a period parallelogram (we take every zero or pole their multiplicity times). Then

$$
a_{1}+\ldots+a_{n} \equiv b_{1}+\ldots+b_{n} \quad(\bmod \Omega)
$$

Further, we will denote by $\omega$ an arbitrary element of the lattice $\Omega$.

One of the main elliptic functions is the Weierstrass $\mathfrak{P}$-function

$$
\mathfrak{P}(z)=\frac{1}{z^{2}}+\sum^{\prime}\left[\frac{1}{(z-\omega)^{2}}-\frac{1}{\omega^{2}}\right]=\frac{1}{z^{2}}+\frac{g_{2}}{20} z^{2}+\frac{g_{3}}{28} z^{4}+\ldots,
$$

where $g_{2}$ and $g_{3}$ are so-called Weierstrass invariants, defined by the equalities

$$
\frac{g_{2}}{60}=\sum^{\prime} \frac{1}{\omega^{4}}, \quad \frac{g_{3}}{140}=\sum^{\prime} \frac{1}{\omega^{6}}
$$

the summation $\sum^{\prime}$ is over all non-zero elements of $\Omega$.

The Weierstrass $\zeta$-function

$$
\zeta(z)=\frac{1}{z}+\sum^{\prime}\left[\frac{1}{z-\omega}+\frac{1}{\omega}+\frac{z}{\omega^{2}}\right]=\frac{1}{z}-\frac{g_{2}}{60} z^{3}-\frac{g_{3}}{140} z^{5}+\ldots
$$

has the properties $\zeta^{\prime}(z)=-\mathfrak{P}(z)$,

$$
\zeta\left(z+\omega_{k}\right)=\zeta(z)+\eta_{k}, \quad k=1,2,
$$

where $\eta_{k}=2 \zeta\left(\omega_{k} / 2\right)$. In every period parallelogram the function has a unique pole with residue 1 .

The following relation is valid

$$
\omega_{2} \eta_{1}-\omega_{1} \eta_{1}=2 \pi i
$$

At last, we need the Weierstrass $\sigma$-function

$$
\sigma(z)=z \prod^{\prime}\left\{\left(1-\frac{z}{\omega}\right) \exp \left(\frac{z}{\omega}+\frac{z^{2}}{2 \omega^{2}}\right)\right\}=z-\frac{g_{2}}{240} z^{5}-\frac{g_{3}}{840} z^{7}+\ldots
$$


It is an odd entire function satisfying

$$
\frac{\sigma^{\prime}(z)}{\sigma(z)}=\zeta(z), \quad \sigma(z+\omega)=\varepsilon \sigma(z) e^{\eta(z+\omega / 2)},
$$

where $\eta=m \eta_{1}+n \eta_{2}$, if $\omega=m \omega_{1}+n \omega_{2} ; \varepsilon=1$ if $\omega / 2$ is a period and $\varepsilon=-1$ otherwise.

Let us write the expansion of a function, connected with the Weierstrass $\zeta$-elliptic functions, at the origin:

$$
\widetilde{\zeta}(z):=\zeta(z)-\frac{1}{z}=-\frac{g_{2}}{60} z^{3}-\frac{g_{3}}{140} z^{5}+\ldots
$$

We will also need the following theorem by Weierstrass. It allows us to recover elliptic function by its zeroes and poles up to a multiplicative constant.

Theorem 3. Every elliptic function $f$ can be represented in the form

$$
f(z)=C \frac{\sigma\left(z-a_{1}\right) \ldots \sigma\left(z-a_{r}\right)}{\sigma\left(z-b_{1}\right) \ldots \sigma\left(z-b_{r}\right)}
$$

where $C \equiv$ const, $a_{1}, \ldots, a_{r}$ is a full system of its zeroes, and $b_{1}, \ldots, b_{r}$ is a full system of its poles, satisfying $a_{1}+\ldots+a_{r}=b_{1}+\ldots+b_{r}$.

The following theorem gives explicit expressions of partial derivatives of the function $\log \sigma\left(z ; \omega_{1}, \omega_{2}\right)$ with respect to $\omega_{1}$ and $\omega_{2}$.

Theorem 4. [13] We have

$$
\begin{aligned}
& \frac{\partial \log \sigma\left(z ; \omega_{1}, \omega_{2}\right)}{\partial \omega_{1}}=\frac{1}{2 \pi i}\left[\frac{1}{2} \omega_{2}\left(\mathfrak{P}(z)-(\zeta(z))^{2}\right)+\eta_{2}(z \zeta(z)-1)+\omega_{2} \frac{g_{2}}{24} z^{2}\right], \\
& \frac{\partial \log \sigma\left(z ; \omega_{1}, \omega_{2}\right)}{\partial \omega_{2}}=-\frac{1}{2 \pi i}\left[\frac{1}{2} \omega_{1}\left(\mathfrak{P}(z)-(\zeta(z))^{2}\right)+\eta_{1}(z \zeta(z)-1)-\omega_{1} \frac{g_{2}}{24} z^{2}\right] .
\end{aligned}
$$

\section{The differential equation for a one-parametric family of el-} liptic functions. Consider a one-parametric family $f(z, t)$ of order $n \geqslant 2$ with periods $\omega_{1}(t)$ and $\omega_{2}(t)$, depending smoothly on a real parameter $t$. Assume that $f(z, t)$ has a unique (up to the period lattice) pole at $z=0$ for every fixed $t$. Then $f^{\prime}(z, t)$ is an elliptic function with the same periods $\omega_{1}(t)$ and $\omega_{2}(t)$. According to Theorem 3 , we have

$$
f^{\prime}(z, t)=\frac{c \prod_{j=0}^{N} \sigma^{m_{j}}\left(z-a_{j}\right)}{\sigma^{n+1}(z)}
$$


where $c=c(t) \neq 0, a_{j}=a_{j}(t)$ are pairwise non-equivalent (with respect to the period lattice $\Omega$ ) points, and the multiplicities $m_{j}$ satisfy the equality $\sum_{j=0}^{N} m_{j}=n+1$.

Differentiating by $z$ and $t$ the periodicity conditions

$$
f\left(z+\omega_{k}(t), t\right)=f(z, t), \quad k=1,2,
$$

we obtain (in more detail, see [13])

Theorem 5. The function

$$
h(z, t):=\frac{\dot{f}(z, t)}{f^{\prime}(z, t)}
$$

satisfies

$$
h\left(z+\omega_{k}(t), t\right)-h(z, t)=-\dot{\omega}_{k}(t), \quad k=1,2 .
$$

Now we will recover the meromorphic function $h(z, t)$ by its singularities for a fixed $t$. For the sake of simplicity, we assume that $\omega_{1} \equiv 1$; this does not restrict generality because the periods $\omega_{k}$ are defined up to a complex non-zero multiplier.

Theorem 6. Under the assumptions above, the family of functions $f(z, t)$ satisfies the equation

$$
\frac{\dot{f}(z, t)}{f^{\prime}(z, t)}=\left.\frac{1}{c} \sum_{k=1}^{N} \frac{\dot{A}_{k}}{\left(m_{k}-1\right) !} \frac{\partial^{m_{k}-1} \widetilde{G}_{k}(\xi)\left[\zeta(\xi)-\zeta(\xi-z)-\eta_{1} z\right]}{\partial \xi^{m_{k}-1}}\right|_{\xi=a_{k}}
$$

where $a_{k}=a_{k}(t), A_{k}=A_{k}(t), \eta_{1}=\eta_{1}(t)$ is defined by (5), and

$$
\widetilde{G}_{k}(z):=G_{k}(z)\left(\frac{z-a_{k}}{\sigma\left(z-a_{k}\right)}\right)^{m_{k}} .
$$

Proof. Let us write the Taylor expansion of $f(z, t)$ at $a_{k}$ :

$$
f(z, t)=A_{k}+B_{k}\left(z-a_{k}\right)^{m_{k}+1}+\ldots
$$

where

$$
B_{k}=\frac{f^{\left(m_{k}+1\right)}\left(a_{k}, t\right)}{\left(m_{k}+1\right) !}
$$

We have

$$
f^{\prime}(z, t)=\frac{c}{G_{k}(z)}\left(\left(z-a_{k}\right)^{m_{k}}+\ldots\right)
$$


where

$$
G_{k}(z)=\frac{\sigma^{n+1}(z)}{\prod_{j=0, j \neq k}^{N} \sigma^{m_{j}}\left(z-a_{j}\right)}
$$

therefore,

$$
B_{k}=\frac{c}{\left(m_{k}+1\right) G_{k}\left(a_{k}\right)} .
$$

From (11) it follows that

$$
\begin{gathered}
f^{\prime}(z, t)=\left(m_{k}+1\right) B_{k}\left(z-a_{k}\right)^{m_{k}}+\ldots, \\
\dot{f}(z, t)=\dot{A}_{k}-\left(m_{k}+1\right) \dot{a}_{k} B_{k}\left(z-a_{k}\right)^{m_{k}}+\ldots,
\end{gathered}
$$

and this implies

$$
\begin{aligned}
& \frac{\dot{f}(z, t)}{f^{\prime}(z, t)}=\frac{\dot{A}_{k}-\left(m_{k}+1\right) \dot{a}_{k} B_{k}\left(z-a_{k}\right)^{m_{k}}+\ldots}{c\left(\left(z-a_{k}\right)^{m_{k}}+\ldots\right)} G_{k}(z) \\
&=\frac{\dot{A}_{k}}{c} \frac{P_{k, m_{k}-1}(z)}{\left(z-a_{k}\right)^{m_{k}}}+O(1), \quad z \rightarrow a_{k}(t),
\end{aligned}
$$

where $P_{k, m_{k}-1}(z)$ is the Taylor polynomial for $\widetilde{G}_{k}(z)$ of degree $\left(m_{k}-1\right)$ at the point $a_{k}$.

From (16) we have

$$
h(z, t)=\sum_{k=1}^{N} \frac{\dot{A}_{k}}{c} \sum_{s=0}^{m_{k}-1} \frac{\widetilde{G}_{k}^{(s)}\left(a_{k}\right)}{s !\left(z-a_{k}\right)^{m_{k}-s}}+O(1), \quad z \rightarrow a_{k} .
$$

Now we rewrite (17) in the form

$$
\begin{aligned}
h(z, t)=\sum_{k=1}^{N} \frac{\dot{A}_{k}}{c} & \sum_{s=0}^{m_{k}-1}(-1)^{m_{k}-s-1} \times \\
& \times \frac{\widetilde{G}_{k}^{(s)}\left(a_{k}\right)\left(\left(z-a_{k}\right)^{-1}\right)^{\left(m_{k}-s-1\right)}}{s !\left(m_{k}-s-1\right) !}+O(1), \quad z \rightarrow a_{k} .
\end{aligned}
$$

The function $\dot{f}(z, t)$ has a pole of order at most $n$ at the point $z=0$, and the order of the pole $f^{\prime}(z, t)$ is equal to $(n+1)$. Therefore, $h(z, t)$ vanishes at $z=0$.

The function 


$$
\begin{aligned}
G(z, t):=h(z, t)-\frac{1}{c} \sum_{k=1}^{N} \dot{A}_{k} \sum_{s=0}^{m_{k}-1}(-1)^{m_{k}-s-1} & \times \\
& \times \frac{\widetilde{G}_{k}^{(s)}\left(a_{k}\right) \zeta^{\left(m_{k}-s-1\right)}\left(z-a_{k}\right)}{s !\left(m_{k}-s-1\right) !}
\end{aligned}
$$

has only removable singularities at all points of the lattice $\Omega$, and it is holomorphic at others points of the complex plane. Consequently, it can be extended, as a holomorphic function, to the whole plane $\mathbb{C}$.

By (5) and (9), taking into account doubly-periodicity of the Weierstrass $\zeta$-function, we have

$$
G\left(z+\omega_{k}, t\right)-G(z, t)=-\dot{\omega}_{k}-\frac{\eta_{k}}{c} \sum_{k=1}^{N} \dot{A}_{k} \frac{\widetilde{G}_{k}^{\left(m_{k}-1\right)}\left(a_{k}\right)}{\left(m_{k}-1\right) !} .
$$

From (19) we see that at infinity $G$ grows not faster than a linear function; therefore,

$$
G(z, t)=\alpha z+\beta
$$

Thus,

$$
\begin{aligned}
h(z, t)=\frac{1}{c} \sum_{k=1}^{N} \dot{A}_{k} \sum_{s=0}^{m_{k}-1}(-1)^{m_{k}-s-1} \times & \\
& \times \frac{\widetilde{G}_{k}^{(s)}\left(a_{k}\right) \zeta^{\left(m_{k}-s-1\right)}\left(z-a_{k}\right)}{s !\left(m_{k}-s-1\right) !}+\alpha z+\beta .
\end{aligned}
$$

From $h(0, t)=0$ we find

$$
\begin{gathered}
\beta=-\frac{1}{c} \sum_{k=1}^{N} \dot{A}_{k} \sum_{s=0}^{m_{k}-1}(-1)^{m_{k}-s-1} \frac{\widetilde{G}_{k}^{(s)}\left(a_{k}\right) \zeta^{\left(m_{k}-s-1\right)}\left(-a_{k}\right)}{s !\left(m_{k}-s-1\right) !}= \\
=\frac{1}{c} \sum_{k=1}^{N} \dot{A}_{k} \sum_{s=0}^{m_{k}-1} \frac{\widetilde{G}_{k}^{(s)}\left(a_{k}\right) \zeta^{\left(m_{k}-s-1\right)}\left(a_{k}\right)}{s !\left(m_{k}-s-1\right) !}= \\
=\left.\frac{1}{c} \sum_{k=1}^{N} \dot{A}_{k} \frac{\left(\widetilde{G}_{k}(z) \zeta(z)\right)^{\left(m_{k}-1\right)}}{\left(m_{k}-1\right) !}\right|_{z=a_{k}} .
\end{gathered}
$$

From (19) it follows that

$$
\alpha \omega_{k}=-\dot{\omega}_{k}-\frac{\eta_{k}}{c} \sum_{j=1}^{N} \dot{A}_{j} \frac{\widetilde{G}_{j}^{\left(m_{j}-1\right)}\left(a_{j}\right)}{\left(m_{j}-1\right) !}, \quad k=1,2 .
$$


Since $\omega_{1}=\omega_{1}(t) \equiv 1$, we obtain from $(22)$

$$
\alpha=-\frac{\eta_{1}}{c} \sum_{k=1}^{N} \dot{A}_{k} \frac{\widetilde{G}_{k}^{\left(m_{k}-1\right)}\left(a_{k}\right)}{\left(m_{k}-1\right) !} .
$$

Finally, from (20), (21), and (23) we deduce (10).

5. System of equations for the parameters. Now we will find a system of ODEs for the parameters $a_{k}=a_{k}(t), \omega_{2}=\omega_{2}(t)$, and $c=c(t)$.

Theorem 7. Let $f(z, t)$ be a smooth one-parametric family of elliptic functions with periods $\omega_{1}=\omega_{1}(t) \equiv 1$ and $\omega_{2}=\omega_{2}(t)$ and let their derivatives have the form (8). Then the critical points $a_{j}=a_{j}(t)$ of $f(z, t)$, the period $\omega_{2}(t)$, and the multiplier $c(t)$ in (8) satisfy

$$
\begin{gathered}
\dot{a}_{k}=\frac{1}{c}\left[\dot{A}_{k}\left(\frac{\widetilde{G}_{k}^{\left(m_{k}\right)}\left(a_{k}\right)}{m_{k} !}-\left.\frac{\partial^{m_{k}-1} \widetilde{G}_{k}(\xi)\left[\zeta(\xi)-\widetilde{\zeta}\left(\xi-a_{k}\right)-\eta_{1} a_{k}\right]}{\left(m_{k}-1\right) ! \partial \xi^{m_{k}-1}}\right|_{\xi=a_{k}}\right)-\right. \\
\left.-\left.\sum_{j=1, j \neq k}^{N} \frac{\dot{A}_{j}}{\left(m_{j}-1\right) !} \frac{\partial^{m_{j}-1}}{\partial \xi^{m_{j}-1}} \widetilde{G}_{j}(\xi)\left[\zeta(\xi)-\zeta\left(\xi-a_{k}\right)-\eta_{1} a_{k}\right]\right|_{\xi=a_{j}}\right], \quad(24) \\
\dot{\omega}_{2}=\frac{2 \pi i}{c} \sum_{k=1}^{N} \dot{A}_{k} \frac{\widetilde{G}_{k}^{\left(m_{k}-1\right)}\left(a_{k}\right)}{\left(m_{k}-1\right) !}, \\
\dot{c}=-c \sum_{j=0}^{N} m_{j}\left[\zeta\left(a_{j}\right) \dot{a}_{j}+\dot{\omega}_{2} \frac{\partial \log \sigma\left(a_{j}\right)}{\partial \omega_{2}}\right]+ \\
+\left.n \sum_{k=1}^{N} \frac{\dot{A}_{k}}{\left(m_{k}-1\right) !} \frac{\partial^{m_{k}-1} \widetilde{G}_{k}(\xi)\left(\mathfrak{P}(\xi)+\eta_{1}\right)}{\partial \xi^{m_{k}-1}}\right|_{\xi=a_{k}}
\end{gathered}
$$

the partial derivative $\partial \log \sigma / \partial \omega_{2}$ in (26) can be found with the help of Theorem 4.

Proof. From (22) and (23) we find

$$
\dot{\omega}_{2}=\frac{\omega_{2} \eta_{1}-\eta_{2}}{c} \sum_{k=1}^{N} \dot{A}_{k} \frac{\widetilde{G}_{k}^{\left(m_{k}-1\right)}\left(a_{k}\right)}{\left(m_{k}-1\right) !},
$$

and, with the help of the equality (6), we obtain (25). 
From (8), (15), and (10) we deduce

$$
\begin{aligned}
& \left(m_{k}+1\right) \dot{a}_{k} B_{k}\left(z-a_{k}\right)^{m_{k}}+\ldots=\dot{A}_{k}-h(z, t) f^{\prime}(z, t)= \\
& =\dot{A}_{k}\left[1-\frac{\left(z-a_{k}\right)^{m_{k}}}{\partial^{m_{k}-1} \widetilde{G}_{k}(\xi)\left[\zeta(\xi)-\zeta(\xi-z)-\eta_{1} z\right]}\right. \\
& \widetilde{G}_{k}(z)\left(m_{k}-1\right) ! \partial \xi^{m_{k}-1} \\
& -\left.\frac{\prod_{j=0}^{N} \sigma^{m_{j}}\left(z-a_{j}\right)}{\sigma^{n+1}(z)} \sum_{j=1, j \neq k}^{N} \frac{\dot{A}_{j} \partial^{m_{j}-1} \widetilde{G}_{j}(\xi)\left[\zeta(\xi)-\zeta(\xi-z)-\eta_{1} z\right]}{\left(m_{j}-1\right) ! \partial \xi^{m_{j}-1}}\right|_{\xi=a_{j}} .
\end{aligned}
$$

Now we will find the coefficient of $\left(z-a_{k}\right)^{m_{k}}$ in the last expression, which is the difference of two expressions, $E_{1}$ and $E_{2}$. For the second expression $E_{2}$ we have

$$
\begin{gathered}
\left.\frac{\prod_{j=0}^{N} \sigma^{m_{j}}\left(z-a_{j}\right)}{\sigma^{n+1}(z)} \sum_{j=1, j \neq k}^{N} \frac{\dot{A}_{j} \partial^{m_{j}-1} \widetilde{G}_{j}(\xi)\left[\zeta(\xi)-\zeta(\xi-z)-\eta_{1} z\right]}{\left(m_{j}-1\right) ! \partial \xi^{m_{j}-1}}\right|_{\xi=a_{j}}= \\
=\left.\sum_{j=1, j \neq k}^{N} \frac{\dot{A}_{j}(t)}{\left(m_{j}-1\right) !} \frac{\dot{A}_{j} \partial^{m_{j}-1} \widetilde{G}_{j}(\xi)\left[\zeta(\xi)-\zeta\left(\xi-a_{k}\right)-\eta_{1} a_{k}\right]}{\left(m_{j}-1\right) ! \partial \xi^{m_{j}-1}}\right|_{\xi=a_{j}} \times \\
\quad \times \frac{\prod_{j=0, j \neq k}^{N} \sigma^{m_{j}}\left(a_{k}-a_{j}\right)}{\sigma^{n+1}\left(a_{k}\right)}\left(z-a_{k}\right)^{m_{k}}+o\left(\left(z-a_{k}\right)^{m_{k}}\right), \quad z \rightarrow a_{k} .
\end{gathered}
$$

Now we need an auxiliary result from [12].

Lemma 1. Let $f$ be $n$ times differentiable at a point $a, f(a) \neq 0$ and $P_{j}$ be the Taylor polynomial for $f$ of degree $j$ at $a$. Then

$$
\begin{gathered}
P_{n}(x)=\left.\frac{(x-a)^{n+1}}{n !} \frac{\partial^{n}}{\partial \xi^{n}}\left(\frac{f(\xi)}{x-\xi}\right)\right|_{\xi=a}, \\
\frac{P_{n-1}(x)}{f(x)}=1-\frac{1}{n !} \frac{f^{(n)}(a)}{f(a)}(x-a)^{n}+o\left((x-a)^{n}\right), \quad x \rightarrow a .
\end{gathered}
$$

For the first expression $E_{1}$ we have

$$
1-\left.\frac{\left(z-a_{k}\right)^{m_{k}}}{\widetilde{G}_{k}(z)\left(m_{k}-1\right) !} \frac{\partial^{m_{k}-1}}{\partial \xi^{m_{k}-1}} \widetilde{G}_{k}(\xi)\left[\zeta(\xi)-\zeta(\xi-z)-\eta_{1} z\right]\right|_{\xi=a_{k}}=
$$




$$
\begin{gathered}
=1-\left.\frac{\left(z-a_{k}\right)^{m_{k}}}{\widetilde{G}_{k}(z)\left(m_{k}-1\right) !} \frac{\partial^{m_{k}-1}}{\partial \xi^{m_{k}-1}} \widetilde{G}_{k}(\xi)(z-\xi)^{-1}\right|_{\xi=a_{k}}- \\
-\left.\frac{\left(z-a_{k}\right)^{m_{k}}}{\widetilde{G}_{k}\left(a_{k}\right)\left(m_{k}-1\right) !} \frac{\partial^{m_{k}-1}}{\partial \xi^{m_{k}-1}} \widetilde{G}_{k}(\xi)\left[\zeta(\xi)-\widetilde{\zeta}\left(\xi-a_{k}\right)-\eta_{1} a_{k}\right]\right|_{\substack{+o a_{k} \\
+o}}\left(\left(z-a_{k}\right)^{m_{k}}\right),
\end{gathered}
$$

therefore, with the help of Lemma 1 , the term with $\left(z-a_{k}\right)^{m_{k}}$ can be written as

$$
\frac{\left(z-a_{k}\right)^{m_{k}}}{\widetilde{G}_{k}\left(a_{k}\right)}\left[\frac{\widetilde{G}_{k}^{\left(m_{k}\right)}\left(a_{k}\right)}{m_{k} !}-\left.\frac{\partial^{m_{k}-1} \widetilde{G}_{k}(\xi)\left[\zeta(\xi)-\widetilde{\zeta}\left(\xi-a_{k}\right)-\eta_{1} a_{k}\right]}{\left(m_{k}-1\right) ! \partial \xi^{m_{k}-1}}\right|_{\xi=a_{k}}\right]
$$

as $z \rightarrow a_{k}$, where $\widetilde{\zeta}(z)=\zeta(z)-1 / z$. Thus, from (27) we deduce (24).

Finally, we will find a differential equation for $c$. From (8) and (10) we obtain the following expansion at $z=0$ :

$$
\begin{aligned}
\dot{f}(z, t)=(-1)^{n} \sum_{k=1}^{N} \frac{\dot{A}_{k}}{\left(m_{k}-1\right) !} \frac{\partial^{m_{k}-1}}{\partial \xi^{m_{k}-1}} & \left.\widetilde{G}_{k}(\xi)\left[\mathfrak{P}(\xi)+\eta_{1}\right]\right|_{\xi=a_{k}} \times \\
& \times \prod_{j=0}^{N} \sigma^{m_{j}}\left(a_{j}\right) \frac{1}{z^{n}}+O\left(\frac{1}{z^{n-1}}\right) .
\end{aligned}
$$

Thus,

$$
\begin{array}{r}
\left.\dot{f}^{\prime}(z, t) \sim(-1)^{n+1} n \sum_{k=1}^{N} \frac{\dot{A}_{k}}{\left(m_{k}-1\right) !} \frac{\partial^{m_{k}-1}}{\partial \xi^{m_{k}-1}} \widetilde{G}_{k}(\xi)\left[\mathfrak{P}(\xi)+\eta_{1}\right]\right|_{\xi=a_{k}} \times \\
\times \prod_{j=0}^{N} \sigma^{m_{j}}\left(a_{j}\right) \frac{1}{z^{n+1}}, \quad z \rightarrow 0 .
\end{array}
$$

On the other hand,

$$
f^{\prime}(z, t)=(-1)^{n+1} c \prod_{k=0}^{N} \sigma^{m_{k}}\left(a_{k}\right) \frac{1}{z^{n+1}}+O\left(\frac{1}{z^{n}}\right),
$$

and, therefore,

$$
\dot{f}^{\prime}(z, t) \sim(-1)^{n+1} \prod_{k=0}^{N} \sigma^{m_{k}}\left(a_{k}\right) \times
$$




$$
\times\left\{\dot{c}+c \sum_{j=0}^{N} m_{j}\left[\zeta\left(a_{j}\right) \dot{a}_{j}+\dot{\omega}_{2} \frac{\partial \log \sigma\left(a_{j}\right)}{\partial \omega_{2}}\right]\right\} \frac{1}{z^{n+1}}, \quad z \rightarrow 0
$$

Now, from (28) and (29) we obtain (26).

Acknowledgment. This work was supported by the Russian Foundation for Basic Research, grant 18-41-160003.

The author is grateful to the anonymous referee for careful reading of the paper and useful remarks.

\section{References}

[1] Akhiezer N.I. Elements of the Theory of Elliptic Functions. Transl. of Mathematical Monographs, vol. 79, American Mathematical Soc., RI, 1990.

[2] Alexandrov I.A. Parametric continuations in the theory of univalent functions. Nauka, Moscow, 1976 (in Russian).

[3] Hurwitz A. Über Riemannsche Flächen mit gegeben Verzweigungpunkten. Math. Ann., 1891, vol. 39, pp. 1-61.

[4] Hurwitz A. Über die Anzahl der Riemannsche Flä̈hen mit gegeben Verzweigungpunkten. Math. Ann., 1902, vol. 55, pp. 53-66.

[5] Lando S.K. Ramified coverings of the two-dimensional sphere and the intersection theory in spaces of meromorphic functions on algebraic curves. Russian Math. Surveys, 2002, vol. 57, no. 3, pp. 463-533. DOI: https: //doi.org/10.1070/RM2002v057n03ABEH000511

[6] Lando S.K., Zvonkin A.K. Graphs on Surfaces and Their Applications. Encyclopaedia Math. Sci., vol. 141, Springer Verlag, Berlin-HeidelbergNew York, 2004. DOI: https://doi.org/10.1007/978-3-540-38361-1

[7] Lloyd E. Riemann surface transformation groups. J. Combin. Theory Ser. A, 1972, vol. 13, pp. 17-27.

[8] Mednykh A.D. Nonequivalent coverings of Riemann surfaces with a prescribed ramification type. Sib. Math. J., 1984, vol. 25, no. 4, pp. 120-142 (in Russian).

[9] Mednykh A.D. A new method for counting coverings over manifold with finitely generated fundamental group. Dokl. Math., 2006, vol. 74, no. 1, pp. 498-502. DOI: https://doi.org/10.1134/S1064562406040089

[10] Nasyrov, S.R., Geometric Problems of the Theory of Ramified Coverings of Riemann Surfaces. Magarif, Kazan, 2008. 
[11] Nasyrov S.R. Determination of the polynomial uniformizing a given compact Riemann surface. Math. Notes, 2012, vol. 91, no. 3-4, pp. 558-567. DOI: https://doi.org/10.1134/S0001434612030303

[12] Nasyrov S.R. Uniformization of Simply-Connected Ramified Coverings of the Sphere by Rational Functions. Lobachevskii J. Math., 2018, vol. 39, no. 2, pp. 252-258. DOI: https://doi.org/10.1134/S1995080218020208

[13] Nasyrov S.R. Uniformization of one-parametric families of complex tori. Russian Math. (Iz. VUZ), 2017, vol. 61, no. 8, pp. 36-45. DOI: https: //doi.org/10.3103/S1066369X17080047

[14] Weyl H. Über das Hurwitzsche Problem der Bestimmung der Anzahl Riemannscher Flächen von gegebener Verzweigungsart, Comment. Math. Helv., 1931, vol. 3, pp. 103-113.

Received August 30, 2018.

In revised form, November 26, 2018.

Accepted November 29, 2018.

Published online December 12, 2018.

Kazan Federal University

35 Kremlevskaya str., Kazan 420008, Russia

E-mail: snasyrov@kpfu.ru 